\title{
Rv3131, a gene encoding nitroreductase, is essential for metronidazole activation in Mycobacterium tuberculosis under hypoxic condition
}

\section{Wenzhu Dong}

First People's Hospital of Ziyang

Jin Shi

Beijing Chest Hospital

Ping Chu

Beijing Children's Hospital

Rongmei Liu

Beijing Chest Hospital

\section{Shu'an Wen}

Beijing Chest Hospital

Tingting Zhang

Beijing Chest Hospital

\section{Yu Pang}

Beijing Chest Hospital

Jie Lu ( $\square$ lujiebch@163.com )

Beijing Chest Hospital

\section{Research}

Keywords: metronidazole, MTZ, Mtb, Cys279Ser

Posted Date: September 14th, 2020

DOI: https://doi.org/10.21203/rs.3.rs-60221/v1

License: (c) (i) This work is licensed under a Creative Commons Attribution 4.0 International License. Read Full License 


\section{Abstract}

Objectives

The impressive potency of metronidazole (MTZ) against anaerobic bacteria indicates the potential for killing anaerobic Mtb. However, how MTZ is activated in Mtb still remains unknown. We aimed to characterize the endogenous nitroreductase responsible for MTZ activation in anaerobic Mtb.

Methods

The minimal inhibitory concentrations (MICs) of Mtb isolates against MTZ were determined by microplate Alamar Blue assay. Intracellular anti-TB activities of MTZ and pyrazinamide (PZA) were tested in THP-1 cells infected by Mycobacterium tuberculosis (Mtb) H37Rv with a multiplicity of infection (MOI) of 10. The nitroreductase activity of purified wild-type Rv3131 and mutants were measured under anaerobic conditions generated by glucose oxidase/catalase system. Two-tailed unpaired Student's t test was used to compare the difference between various groups.

Results

180 Mtb isolates $(81.8 \%, 180 / 220)$ had MIC values higher than $16 \mu \mathrm{g} / \mathrm{mL}$, and 40 had MIC values of 16 $\mu \mathrm{g} / \mathrm{mL}$, demonstrating high-level resistance to MTZ under aerobic condition. The number of viable bacteria in macrophages treated with MTZ was dramatically decreased by $71.3 \%$ after 5 -day MTZ treatment, indicating significant inhibition of MTZ against anaerobic Mtb. In vitro biochemical analysis demonstrated that Rv3131 exhibited the NADPH oxidase activity under anaerobic condition. The substitutions of Cys75Ser and Cys 279 Ser could maintain $41.7 \%$ and $71.1 \%$ of enzyme activity compared to wild-type protein, respectively.

Conclusions

Our data demonstrate that MTZ has more potent efficacy against intracellular Mtb than PZA. Rv3131 is identified as a nitroreductase enzyme in the activation of MTZ, and Cys75 of Rv3131 is the major active residue for nitroreductase activity.

\section{Introduction}

Tuberculosis (TB), caused by the intracellular pathogen, Mycobacterium tuberculosis (Mtb), remains one of the deadliest infectious diseases, accounting for 1.45 million deaths in the year 2018. ${ }^{1,2}$ In the past decades, great progress has achieved in combating tuberculosis due to the implementation of effective strategies, whereas the enormous burden of latent TB infection (LTBI) poses a major obstacle to TB control. $^{3}$ Individuals with LTBI are assumed to have a $10 \%$ lifetime risk of developing active TB diseases, ${ }^{3}$ and this risk is significantly increased for immune-competent persons, such as HIV infection and administration of immunosuppressive agents. ${ }^{4}$ Given that one third of the world population are latently 
infected with Mtb, the potential progression from LTBI to disease state highlights the urgent need to improve management of latent infections. ${ }^{3}$

Preventive treatment has been considered as an effective option to prevent the activation of TB in human. ${ }^{5}$ The World Health Organization recommends 6 or 9 months of isoniazid monotherapy, 3 months of rifampicin plus isoniazid, or 3 months of weekly rifapentine plus isoniazid as the standard treatment of LTBI. ${ }^{6}$ A number of randomized clinical trials have confirmed that preventive treatment could bring benefits to individuals infected with drug susceptible bacteria. ${ }^{7-9}$ As a consequence, the delivery of preventive therapy for high-risk individuals with LTBI is employed as a core strategy of TB control programmes in high-income countries for half a century. ${ }^{4}$ Although these anti-TB drugs have activity against both intracellular and extracellular Mtb, their preference to kill replicating bacilli compels clinicians to use the protracted regimen for sterilizing nonreplicating mycobacteria. ${ }^{10}$ Compliance with the long treatment course of chemotherapy becomes the major challenge for LTBI at high-risk. ${ }^{10}$ Therefore, the effective antimicrobial agent targeting nonreplicating subpopulation of tubercle bacilli is essential for shortening therapy for LTBI.

Metronidazole (MTZ) is a 5-nitroimidazole antibiotic mainly used in the treatment of anaerobic and protozoal infections. ${ }^{11,12}$ The impressive potency of MTZ against anaerobic bacteria indicates the potential for killing Mtb reservoir under anaerobic condition in individuals with LTBI. Previous in vitro experimental studies have demonstrated that MTZ has potent activity against tubercle bacilli under anaerobic condition, whereas has no efficacy under aerobic condition. ${ }^{13,14}$ In the nonhuman primate model, MTZ prevents reactivation of latent Mtb infection, indicating its in vivo role in targeting Mtb within anaerobic condition. ${ }^{15}$ Although there is strong evidence for the role of MTZ in affecting anaerobic bacilli, its mechanism of action has not yet been elucidated in Mtb considering that MTZ must be catalyzed to a nitro free radical form with endogenous nitroreductase. To provide insights into how MTZ is activated in anaerobic Mtb bacilli, we have firstly characterized the nitroreductase gene (Rv3131), and identified the key residue conferring the activation of MTZ in Mtb.

\section{Materials And Methods}

\section{Bacterial strains and growth media}

Mtb H37Rv (ATCC 27294) and Mycobacterium smegmatis (Msm) mc ${ }^{2} 155$ seed-lot stocks were subculture at $37^{\circ} \mathrm{C}$ in Middlebrook $7 \mathrm{H} 9$ broth (BD Difco) supplemented with $10 \%$ oleic acid-albumindextrose-catalase (OADC), or on Middlebrook 7H10 agar (BD Difco) supplemented with $10 \%$ oleic acidalbumin-dexrose-catalase (OADC). In addition, a total of 220 drug-resistant isolates were included from the National Tuberculosis Clinical Laboratory, Beijing Chest Hospital in China. All clinical Mtb isolates were grown on Middlebrook $7 \mathrm{H} 10$ agar supplemented with 10\% OADC for in vitro drug susceptibility analysis. Escherichia coli DH5a was employed for plasmid construction and propagation. Recombinant Mtb Rv3131 was expressed in E. coli BL21(DE3). All E. coli strains were sub-cultured on LB agar or LB 
broth. Kanamycin was supplemented in the growth medium at a concentration of $50 \mu \mathrm{g} / \mathrm{mL}$ for appropriate selection purpose.

\section{Minimal inhibitory concentration}

The minimal inhibitory concentrations (MICs) of Mtb isolates against MTZ were determined by using a microplate Alamar Blue assay as reported previously. ${ }^{16} \mathrm{MTZ}$ was dissolved in dimethyl sulfoxide to a stock concentration of $2.56 \mathrm{mg} / \mathrm{mL}$. The fresh sub-cultured mycobacterial clones were harvested from the surface of Middlebrook 7H10 agar, and the turbidity of cultures was adjusted to $1.0 \mathrm{McFarland}$ standard. Then 1.0 McFarland cell suspension was diluted to 1:20 in Middlebrook 7H9 broth supplemented with $10 \%$ OADC for inoculum. Serial twofold dilutions of MTZ in $100 \mu \mathrm{L}$ of $7 \mathrm{H} 9$ medium were prepared directly in 96-well plate at concentration of 0.032 to $32 \mu \mathrm{g} / \mathrm{mL}$. $100 \mu \mathrm{L}$ of this inoculum was pipetted into the wells of the prepared 96-well plate. After 7-days of incubation at $37^{\circ} \mathrm{C}, 70 \mu \mathrm{L}$ of fresh Alamar Blue solution was pipetted to each well and incubated for additional 24 hours at $37^{\circ} \mathrm{C}$. A pink color was interpreted as bacterial growth, whereas a blue color was scored as no bacterial growth. The MIC was determined as the lowest drug concentration of antimicrobial agent that prevented a color change from blue to pink. Mtb H37Rv (ATCC 27249) was conducted in all runs as a quality control.

\section{Intracellular anti-TB activity in macrophages}

Human THP- 1 monocytic cells $\left(5 \times 10^{5}\right.$ cells $\left./ \mathrm{mL}\right)$ were treated with $100 \mathrm{nM}$ of phorbol myristate acetate (PMA) in a culture flask for $24 \mathrm{~h}$ for differentiation of macrophages. The macrophages were then inoculated with log-phase mycobacterial cells for $2 \mathrm{~h}$ at a multiplicity of infection (MOI) of 10, washed three times with phosphate-buffered saline (PBS) to remove the non-infecting bacteria, and cultured in fresh medium containing MTZ or pyrazinamide (PZA). At various time points post infection, macrophage cells were washed with PBS three times and harvested for Colony-Forming Unit (CFU) counting. The cells were lysed using $0.025 \%(\mathrm{v} / \mathrm{v})$ Sodium dodecyl sulfate (SDS). The viable bacteria were counted on $7 \mathrm{H} 10$ agar plates to assess the potency of antimicrobial agents against intracellular mycobacteria. ${ }^{17}$

\section{Bioinformatic analysis}

In order to find the enzyme involving MTZ activation in Mtb, we utilized BLAST (https://blast.ncbi.nlm.nih.gov/Blast.cgi) to search Mtb proteins in the swissprot database, using the protein sequence of NADPH nitroreductase RdxA in Helicobacter pylori as the query sequence (UniProt accession No. 025608).

The structure modeling was carried out by SWISS-MODEL server (https://www.swissmodel.expasy.org/), using Protein Data Bank (PDB) (http://www.rcsb.org/pdb/) file 2YMV as the template. ${ }^{18}$ Sequence alignments were conducted using clustalO ${ }^{19}$ and the figure was generated by ESPript. ${ }^{20}$

\section{Expression and purification of recombinant Rv3131 protein}


The Rv3131 gene was amplified from Mtb H37Rv genomic DNA with the following primer set: forward, 5'CATATGACCGCAGCCGTTGA-3' (Nde I) and reverse, 5'-AAGCTTGCACCGTTGTCGCA-3' (Hind III). In addition, the Rv3131 mutants with point mutations were chemically synthesized.

The $R v 3131$ fragments were inserted into the pET28b (+) vector (Novagen). Then, $R v 3137$-inserted $\mathrm{pET} 28 \mathrm{~b}(+)$ was transformed into E. coli BL21 (DE3) for protein expression. E. coli BL21 harboring Rv3131-inserted pET28b (+) was induced with $100 \mu \mathrm{M}$ isopropyl $\beta$-D-1-thiogalactopyranoside (IPTG) for 2 h. After cell lysis using sonication, recombinant Rv3131 was purified as described previously, ${ }^{21}$ and stored at $-70^{\circ} \mathrm{C}$ until use. The purity of the recombinant protein was confirmed by Western blot with an anti-histidine antibody (mAB12698s; Cell Signaling). The concentration of the recombinant protein was analysed with the BCA protein assay method according to the manufacturer's instructions (Solarbio, China).

\section{Measurement of Enzyme Activities}

A glucose oxidase/catalase system was employed to generate anaerobic conditions for determining the enzyme activity of Rv3131. Briefly, the standard assay mixture contained $25 \mathrm{mM}$ glucose, $30 \mathrm{units} / \mathrm{mL}$ glucose oxidase, and 30 units/mL catalase in $10 \mathrm{mM}$ Tris-HCl pH 7.5 buffer for generation of anaerobic conditions, and $300 \mu \mathrm{M}$ of NADPH as substrate of Rv3131. The concentration of MTZ in the reaction mixture ranged from $0 \mu \mathrm{M}$ to $150 \mu \mathrm{M}$. After $10 \mathrm{~min}$ of preincubation at $23^{\circ} \mathrm{C}$ for glucose oxidase/catalase reaction, the reaction was initiated by the addition of Rv3131 enzyme. The enzymatic activities were monitored with spectrophotometer at $23^{\circ} \mathrm{C}$, and the decrease in absorbance at $320 \mathrm{~nm}$ was interpreted as the reduction of MTZ. All reactions were repeated in three independent experiments. ${ }^{22}$

\section{Statistical analysis}

The experimental results were expressed as the means \pm standard errors (S.E.). A two-tailed unpaired Student's $t$ test was used to compare the difference between various groups. The difference was declared as significant if $P$ values were less than 0.05 .

\section{Results}

\section{MICs of MTZ against Mtb under aerobic condition}

We firstly assessed in vitro activity of MTZ against clinical MDR- and XDR-TB isolates. Of these 220 clinical isolates comprising 110 MDR-TB and 110 XDR-TB isolates, more than four-fifths isolates (81.8\%, $180 / 220$ ) had MIC values higher than $16 \mu \mathrm{g} / \mathrm{mL}$, and the other 40 had MIC values of $16 \mu \mathrm{g} / \mathrm{mL}$, demonstrating high-level resistance to MTZ under aerobic condition (Figure 1).

\section{Intracellular activity of MTZ against Mtb under anaerobic condition}

In one set of experiments, PZA and MTZ was added after $\mathrm{xx} h$ of intracellular growth of bacteria, respectively. Our results are illustrated in Figure 2. At a concentration of $10 \mu \mathrm{g} / \mathrm{mL}$, PZA showed inhibition 
on the Mtb growth in macrophages, but the growth difference between PZA-treated group and control group (without any treatment) was not significant. However, MTZ significantly inhibited Mtb growth under anaerobic condition, and the number of viable bacteria in macrophages treated with MTZ was dramatically decreased by $71.3 \%$ after treated by MTZ for 5 days. Statistical analysis revealed that the percentage of viable bacteria in macrophages treated with MTZ was significantly lower than that of control group $(\mathrm{P}=0.0065)$.

\section{Rv3131 is a nitroreductase conferring the activation of MTZ in Mtb}

As a prodrug, the intracellular activation of MTZ requires an oxygen-insensitive NADPH nitroreductase. In order to find the possible nitroreductase responsible for MTZ activation, we searched Mtb proteins by BLAST using $H$. pylori RdxA as the query sequence The result showed that a putative nitroreductase Rv3131 with 344 amino acids, was the most similar nitroreductase to H. pylori RdxA. The query coverage, sequence identity and sequence similarity were $97.3 \%, 10.3 \%$ and $23.3 \%$, respectively. Therefore, Rv3131 was selected as a potential candidate enzyme involving in the activation of MTZ in Mtb.

In vitro biochemical analysis demonstrated that Rv3131 exhibited the NADPH oxidase activity under anaerobic condition, and the absorbance of reaction mixture by the end of 30 second reaction was decreased by $34.7 \%$ compared to that in control group. In addition, the absence of glucose oxidase/catalase system resulted in no decrease in the concentration of substrate, indicating that the anaerobic condition was essential for catalytic activity (Figure 3 ).

\section{Structural modeling and sequence alignment}

To further predict the key residue for enzymatic activity, we modeled the structure of Rv3131 using SWISS-MODEL. As shown in Figure 4A, the structure of Rv3131 is formed of two domains. The N-terminal domain consists of a four-stranded antiparallel $\beta$ sheet formed by $\beta 1, \beta 2, \beta 4$ and $\beta 3$, which are surrounded by a helices $\alpha 1, \alpha 2$, and $a 3$. The $C$-terminal domain has a pattern of $\alpha / \beta / \alpha$ sandwitch. It contains a central four-antiparallel $\beta$ sheet $(\beta 5 / \beta 6 / \beta 8 / \beta 7)$, surrounded by helices $\alpha 4$ and $\alpha 7$ on one side and $a 5, a 6$ and $a 8$ on the other side.

In previous study, Cys159 of $H$. pylori RdxA was proved to be required for the nitroreduction of metronidazole, suggesting that cysteines might be the key residues for enzymatic activity of Rv3131. ${ }^{18}$ The result of sequence alignment (Figure 4B) showed that Cys75 and Cys279 were conserved cysteines in Rv3131, which might be the potential key residues for the nitroreductase activity.

\section{Comparison of wild-type and mutant Rv3131 for the nitroreductase activity}

To investigate our hypothesis, the vectors with Cys75Ser and Cys279Ser point mutation in Rv3131 were constructed, respectively. As shown in Figure 5, the mutated Rv3131 showed a significant decrease in the NADPH nitroreductase activity. The Cys75Ser amino acid substitution maintained only $41.7 \%$ of its wild- 
type activity. The substitution of Cys279Ser could maintain $71.1 \%$ of enzyme activity compared to wildtype protein.

\section{Discussion}

MTZ has demonstrated considerable activity against Mtb under hypoxic conditions in in vitro models and early clinical trials. Our evidence firstly demonstrates a key role of Rv3131, a nitroreductase enzyme, in the activation of MTZ. As an endogenous nitroreductase, Rv3131 is speculated to involve the detoxification of nitrogen-containing by-products, thereby providing a protective role against nitrogen stress inside the host. ${ }^{23,24}$ Notably, a previous report revealed that Rv3131 includes two DosR binding sites, suggesting that it is a hypoxic response gene. ${ }^{23}$ Further experiments confirmed that the dormancyspecific Rv3131 highly expresses under in vitro anaerobic conditions, which may be essential for longterm survival within host cells during latent infection. ${ }^{25}$ This may explain why MTZ only produces potent activity against tubercle bacilli under anaerobic condition rather than aerobic condition. During past years a novel series of nitroimidazoles have been developed as new candidates for TB therapy, including pretomanid and delamanid. Similar to MTZ, another native quinone reductase $\mathrm{Ddn}$ is required for activation of these two prodrug nitroimidazoles. ${ }^{26,27}$ In view of its unspecific expression patterns under various survival conditions, pretomanid and delamanid are effective against both active and latent TB infection. Therefore our data confirm that MTZ utilizes a distinct nitroreductase to convert this prodrug into an active form, and the diverse expression patterns of the corresponding activators may play an important role in determining where these prodrug nitroimidazoles could generate promising action against Mtb.

Another interesting of our study was that MTZ has more potent efficacy against intracellular Mtb than PZA. It is well recognized that PZA targets dormant bacilli residing within macrophage. ${ }^{28}$ Nevertheless, experimental evidence on the actual activity of PZA against intracellular Mtb is controversial. ${ }^{29-31}$ In a previous study by Heifets and colleagues, PZA was not active against Mtb in cultured human monocytederived macrophage. ${ }^{29}$ In line with our observation, Salfinger et al. confirmed that PZA had bactericidal and some inhibitory activity against Mtb in cultured human macrophages and in the BACTEC system. ${ }^{30}$ Although the exact reasons remain unclear, we hypothesize that the conflicting results may be due to the strict requirement of acidic $\mathrm{pH}$ for activation of PZA in vivo. However, the phagosome acidification in macrophages could be accelerated by stimulation of gamma interferon (INF- $\gamma$ ) ${ }^{32}$ The human macrophages of different origin may have a great impact on the secretion of INF- $\gamma$, thereby leading to the diversity in phagosome acidification to a pH range where PZA is highly active. In other words, the host response to Mtb infection may be responsible for the variance in clinical response of TB patients to PZAcontaining regimens. In contrast, the activation of MTZ does not need the strict acid environment, which may assure of killing intracellular tubercle bacilli regardless of macrophages' origin. In view of the superior efficacy of MTZ against dormant bacilli, further clinical trials are urgently needed to elucidate our in vitro results. 
The BLAST result revealed that the $\mathrm{C}$-terminal sequence of Rv3131 could produce significant alignments with H. pylori RdxA. Structure modeling showed that the C-terminal domain of Rv3131 had a pattern of a/ $\beta /$ a sandwitch, which was very similar to the structure pattern of $H$. pylori RdxA (PDB file 3QDL). ${ }^{33}$ Interestingly, the Cys279 residue on $\beta 7$ of Rv3131 had a similar location in the $\alpha / \beta / a$ sandwitch pattern with Cys 159 residue on $\beta 7$ of $H$. pylori RdxA. As Cys159 of $H$. pylori RdxA was proved to be required for the nitroreduction of metronidazole, ${ }^{33}$ we previously thought that the Cys279 residue might be the key residue for enzymatic activity of Rv3131. However, the structure of Rv3131 was formed of two domains and sequence alignment showed that there was another conserved cysteine residue, namely Cys75, in Nterminal domain. The analysis of nitroreductase activity and in vitro survival showed that amino acid substitution of Cys75 showed more influence on enzyme activity than amino acid substitution of Cys279, compared to wild-type protein, suggesting that the Cys75 is the predominate residue for catalyzing reduction process of MTZ.

We also acknowledge that there are several obvious limitations in the present study. First, we observed effect of MTZ against intracellular tubercle bacilli by only in vitro experiment. Further in vivo studies are required to determine the anti-TB efficacy of MTZ and MTZ-containing regimens with murine models. Second, the Cys75 of Rv3131 was confirmed as the major active residue for nitroreductase instead of Cys279 located within another domain. Therefore, it is meaningful for full characterization of interaction kinetics between Rv3131 and its substrates by structure data. Nevertheless, our study firstly identified the nitroreductase for activating MTZ in Mtb, which bring a noteworthy potential of regimens with MTZ in treatment of TB patients.

\section{Conclusions}

In conclusion, our data demonstrate that MTZ has more potent efficacy against intracellular Mtb than PZA. Rv3131 is identified as a nitroreductase enzyme in the activation of MTZ, and Cys75 in N-terminal domain of Rv3131 is the major active residue for nitroreductase activity. Further study is urgently needed to confirm the potential efficacy of MTZ against Mtb in murine models and clinical trials.

\section{Declarations}

\section{Ethics approval and consent to participate}

The protocols applied in this study were approved by the Ethics Committee of the Beijing Chest Hospital, Capital Medical University. The methods used in this study were performed in accordance with relevant guidelines and regulations. All patients participating in this study provided written informed consent.

\section{Consent for publication}

This manuscript contained no individual person's data.

\section{Availability of data and materials}


The datasets used and/or analyzed during the current study are available from the corresponding author on reasonable request.

\section{Competing interests section}

The authors declare that they have no conflicts of interest.

\section{Funding}

This work was supported by National Natural Science Foundation of China (81401739), Beijing Advanced Innovation Center for Big Data-Based Precision Medicine (BHME-201804), the Beijing Talents Foundation (2017000021223ZK39).

\section{Authors' Contributions}

Jie Lu and Yu Pang designed the studies and drafted the article. Wenzhu Dong, Jin Shi, Shu'an Wen and Tingting Zhang undertook the experimental work. Wenzhu Dong, Jin Shi and Ping Chu analyzed the data and contributed to figure and manuscript preparation. All the authors approved the final version of the manuscript.

\section{Acknowledgments}

The authors would like to thank members of the National Clinical Laboratory on Tuberculosis at Beijing Chest Hospital, Capital Medical University for their technical assistance.

\section{References}

1. WHO. Global tuberculosis report 2019. Geneva: WHO 2019.

2. Zumla A, George A, Sharma V et al. The WHO 2014 global tuberculosis report-further to go. Lancet Glob Health 2015; 3: e10-2.

3. Churchyard GJ, Swindells S. Controlling latent TB tuberculosis infection in high-burden countries: A neglected strategy to end TB. PLoS Med 2019; 16: e1002787.

4. Chee CBE, Reves R, Zhang $Y$ et al. Latent tuberculosis infection: Opportunities and challenges. Respirology 2018; 23: 893-900.

5. Getahun H, Matteelli A, Abubakar I et al. Management of latent Mycobacterium tuberculosis infection: WHO guidelines for low tuberculosis burden countries. Eur Respir J 2015; 46: 1563-76.

6. WHO. Guidelines on the management of latent tuberculosis infection. Geneva: WHO 2015.

7. Schechter M, Zajdenverg R, Falco $G$ et al. Weekly rifapentine/isoniazid or daily rifampin/pyrazinamide for latent tuberculosis in household contacts. Am J Respir Crit Care Med 2006; 173: 922-6.

8. Ziakas PD, Mylonakis E. 4 months of rifampin compared with 9 months of isoniazid for the management of latent tuberculosis infection: a meta-analysis and cost-effectiveness study that 
focuses on compliance and liver toxicity. Clin Infect Dis 2009; 49: 1883-9.

9. Doan TN, Fox GJ, Meehan MT et al. Cost-effectiveness of 3 months of weekly rifapentine and isoniazid compared with other standard treatment regimens for latent tuberculosis infection: a decision analysis study. J Antimicrob Chemother 2019; 74: 218-27.

10. Connolly LE, Edelstein PH, Ramakrishnan L. Why is long-term therapy required to cure tuberculosis? PLoS Med 2007; 4: e120.

11. Jenks PJ, Ferrero RL, Labigne A. The role of the rdxA gene in the evolution of metronidazole resistance in Helicobacter pylori. J Antimicrob Chemother 1999; 43: 753-8.

12. Lofmark S, Edlund C, Nord CE. Metronidazole is still the drug of choice for treatment of anaerobic infections. Clin Infect Dis 2010; 50 Suppl 1: S16-23.

13. Wayne LG, Sramek HA. Metronidazole is bactericidal to dormant cells of Mycobacterium tuberculosis. Antimicrob Agents Chemother 1994; 38: 2054-8.

14. Klinkenberg LG, Sutherland LA, Bishai WR et al. Metronidazole lacks activity against Mycobacterium tuberculosis in an in vivo hypoxic granuloma model of latency. $J$ Infect Dis 2008; 198: 275-83.

15. Lin PL, Dartois V, Johnston PJ et al. Metronidazole prevents reactivation of latent Mycobacterium tuberculosis infection in macaques. Proc Natl Acad Sci U S A 2012; 109: 14188-93.

16. Zong Z, Jing W, Shi J et al. Comparison of In Vitro Activity and MIC Distributions between the Novel Oxazolidinone Delpazolid and Linezolid against Multidrug-Resistant and Extensively Drug-Resistant Mycobacterium tuberculosis in China. Antimicrob Agents Chemother 2018; 62.

17. Mor N, Vanderkolk J, Heifets L. Inhibitory and bactericidal activities of levofloxacin against Mycobacterium tuberculosis in vitro and in human macrophages. Antimicrob Agents Chemother 1994; 38: 1161-4.

18. Chauviac FX, Bommer M, Yan J et al. Crystal structure of reduced MsAcg, a putative nitroreductase from Mycobacterium smegmatis and a close homologue of Mycobacterium tuberculosis Acg. J Biol Chem 2012; 287: 44372-83.

19. Madeira F, Park YM, Lee J et al. The EMBL-EBI search and sequence analysis tools APIs in 2019. Nucleic Acids Res 2019; 47: W636-W41.

20. Robert $X$, Gouet P. Deciphering key features in protein structures with the new ENDscript server. Nucleic Acids Res 2014; 42: W320-4.

21. Lu J, Wang X, Xia B et al. Solution structure of Apo-YjaB from Escherichia coli. Proteins 2009; 76 : 261-5.

22. Olekhnovich IN, Goodwin A, Hoffman PS. Characterization of the NAD(P)H oxidase and metronidazole reductase activities of the RdxA nitroreductase of Helicobacter pylori. FEBS J 2009; 276: 3354-64.

23. Sassetti CM, Rubin EJ. Genetic requirements for mycobacterial survival during infection. Proc Nat/ Acad Sci U S A 2003; 100: 12989-94. 
24. Kwon KW, Kim WS, Kim H et al. Novel vaccine potential of Rv3131, a DosR regulon-encoded putative nitroreductase, against hyper-virulent Mycobacterium tuberculosis strain K. Sci Rep 2017; 7: 44151.

25. Voskuil MI, Schnappinger D, Visconti KC et al. Inhibition of respiration by nitric oxide induces a Mycobacterium tuberculosis dormancy program. J Exp Med 2003; 198: 705-13.

26. Awasthi D, Freundlich JS. Antimycobacterial Metabolism: Illuminating Mycobacterium tuberculosis Biology and Drug Discovery. Trends Microbio/ 2017; 25: 756-67.

27. Wen S, Jing W, Zhang $T$ et al. Comparison of in vitro activity of the nitroimidazoles delamanid and pretomanid against multidrug-resistant and extensively drug-resistant tuberculosis. Eur J Clin Microbiol Infect Dis 2019; 38: 1293-6.

28. Njire $\mathrm{M}$, Tan $\mathrm{Y}$, Mugweru $\mathrm{J}$ et al. Pyrazinamide resistance in Mycobacterium tuberculosis: Review and update. Adv Med Sci 2016; 61: 63-71.

29. Heifets $L$, Higgins M, Simon B. Pyrazinamide is not active against Mycobacterium tuberculosis residing in cultured human monocyte-derived macrophages. Int J Tuberc Lung Dis 2000; 4: 491-5.

30. Salfinger M, Crowle AJ, Reller LB. Pyrazinamide and pyrazinoic acid activity against tubercle bacilli in cultured human macrophages and in the BACTEC system. J Infect Dis 1990; 162: 201-7.

31. Ahmad Z, Fraig MM, Bisson GP et al. Dose-dependent activity of pyrazinamide in animal models of intracellular and extracellular tuberculosis infections. Antimicrob Agents Chemother 2011; 55: 152732.

32. Vandal $\mathrm{OH}$, Pierini LM, Schnappinger $\mathrm{D}$ et al. A membrane protein preserves intrabacterial $\mathrm{pH}$ in intraphagosomal Mycobacterium tuberculosis. Nat Med 2008; 14: 849-54.

33. Martinez-Julvez M, Rojas AL, Olekhnovich I et al. Structure of RdxA-an oxygen-insensitive nitroreductase essential for metronidazole activation in Helicobacter pylori. FEBS J 2012; 279: 430617.

\section{Figures}




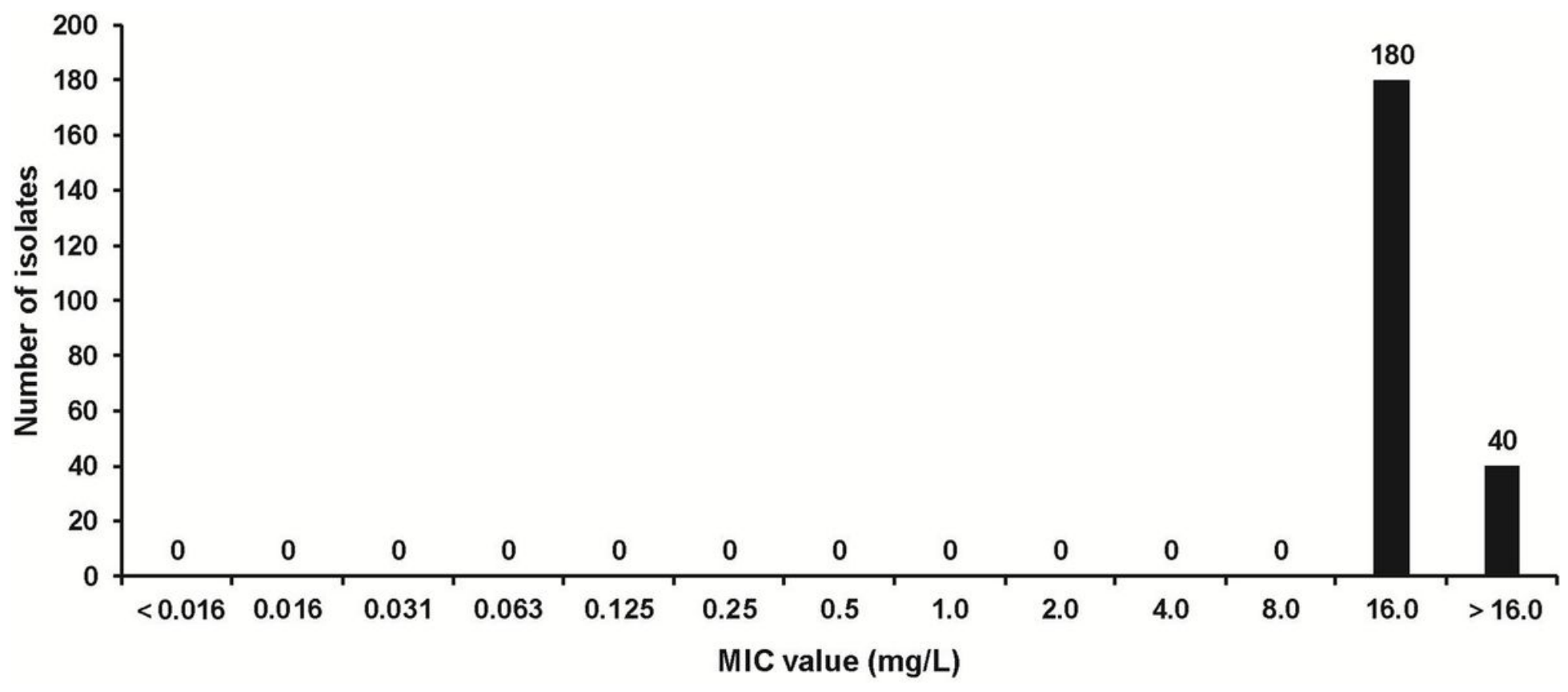

Figure 1

MTZ significantly inhibited Mtb growth in macrophages.

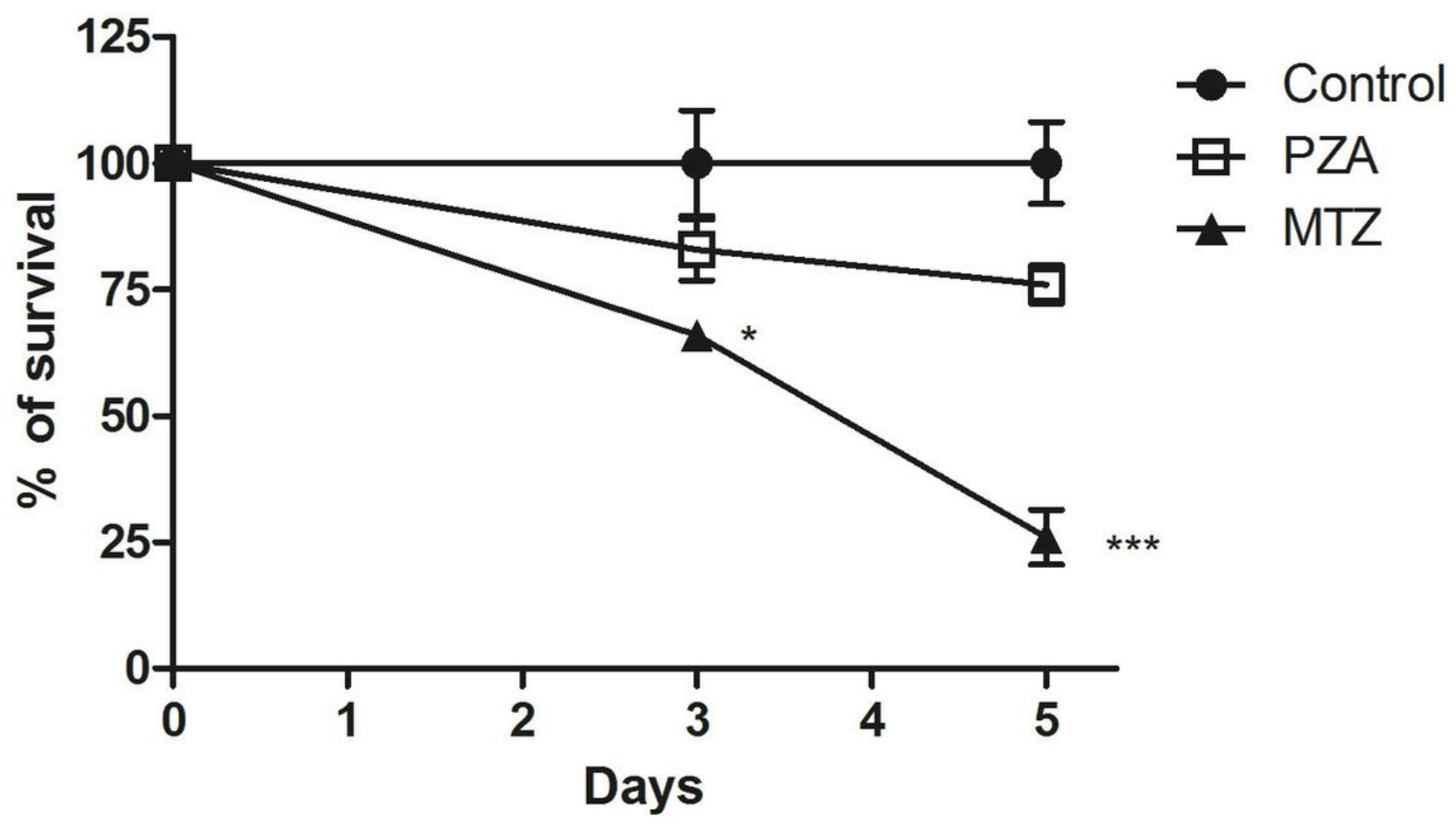

Figure 2

Intracellular activity of MTZ against Mtb under anaerobic condition. 


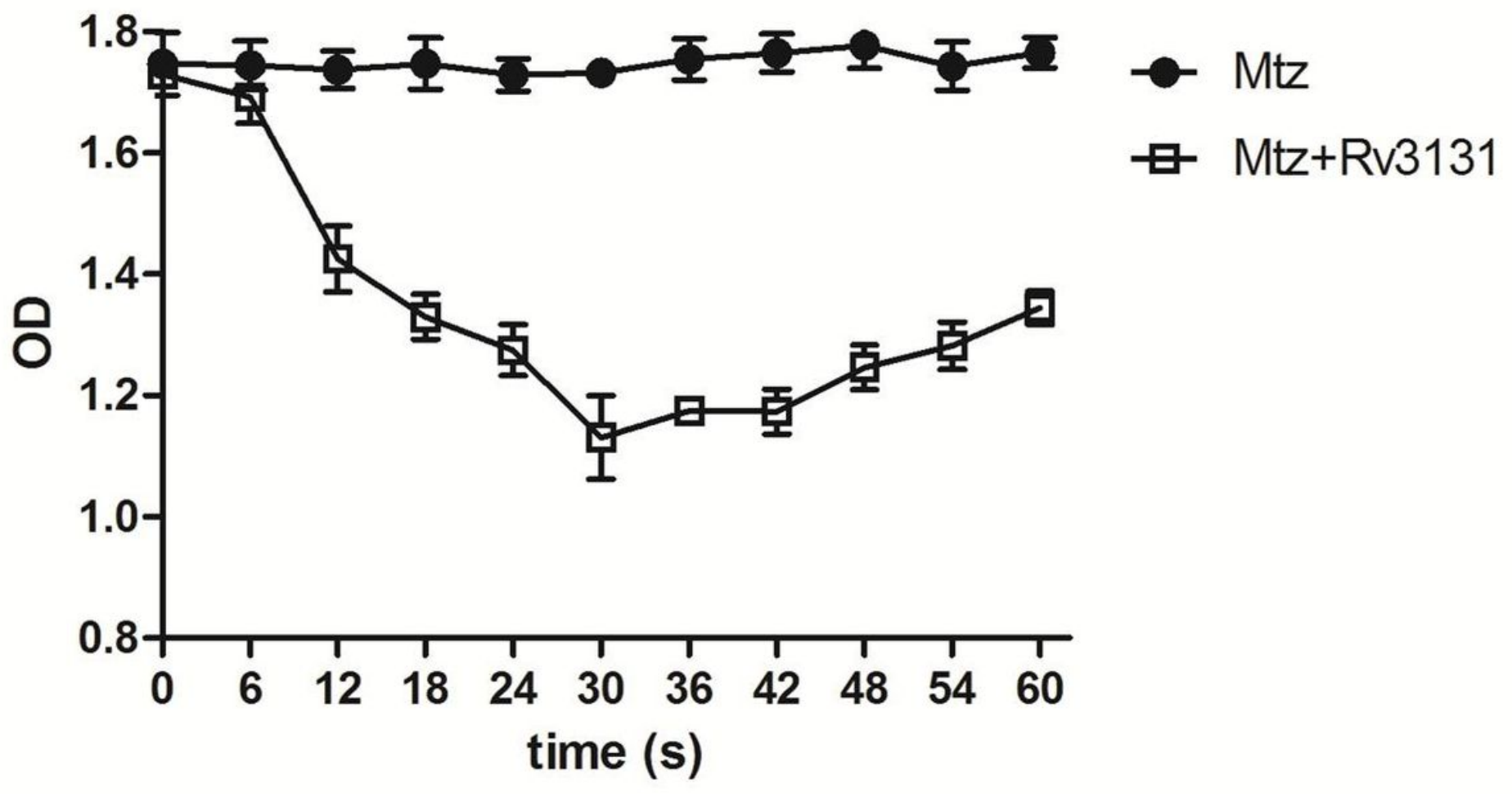

Figure 3

In vitro biochemical analysis for the nitroreductase activity of Rv3131. 
A

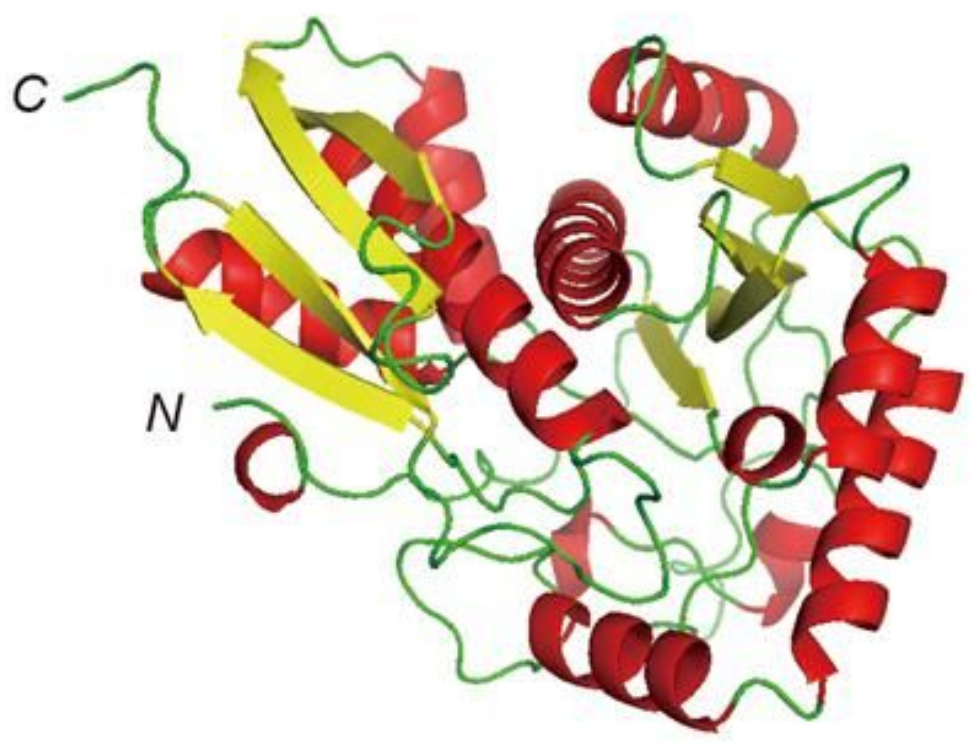

B

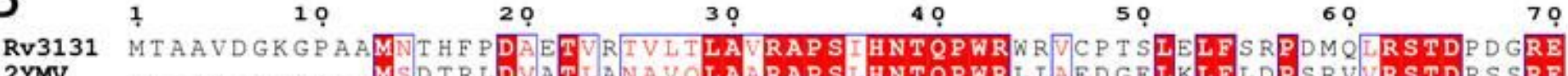

2YMV $\ldots \ldots \ldots$ MSD TRLDVATLANAVOLARAPSLHNTOPWRLIAEDGELKLFLDESRVVRSTDRSSRE

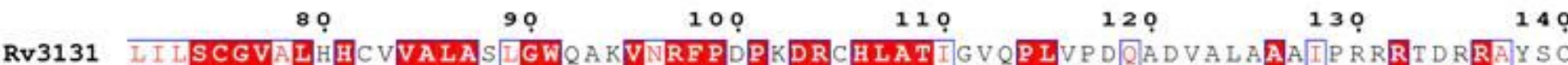
2YMV AVMSCGVLLDHLRVALAAAGWDTEVRFPNBNDRDHLATLSFRPLQFVIEGHRKRADAILARRTDLPMS
150
160
170
180
190
200

Rv3131 WPVPGGDIALMAARAARGGVMLRQVSALDRMKAIVAQAVL̈DHVTDEEYLREL. . . . TIWSGRYGSVAGV 2YMV AYVDWDAFETLLRARLGDGPUHMDTLGEDVREEVAEAAALTESLRLYDEAYHSELAWWTTPFATEDGIPQ

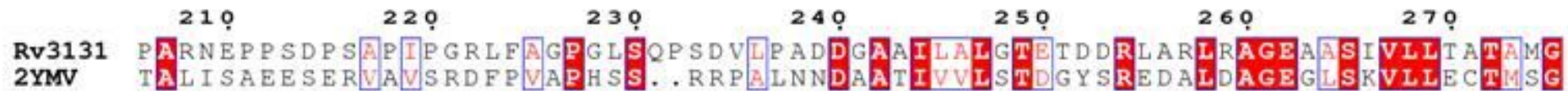

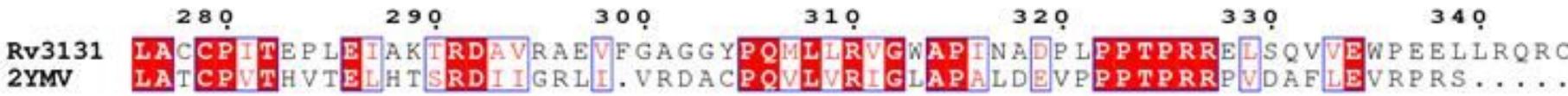

\section{Figure 4}

Structural model of Rv3131 (A) and sequence alignment between Rv3131 and the modeling template (B). 


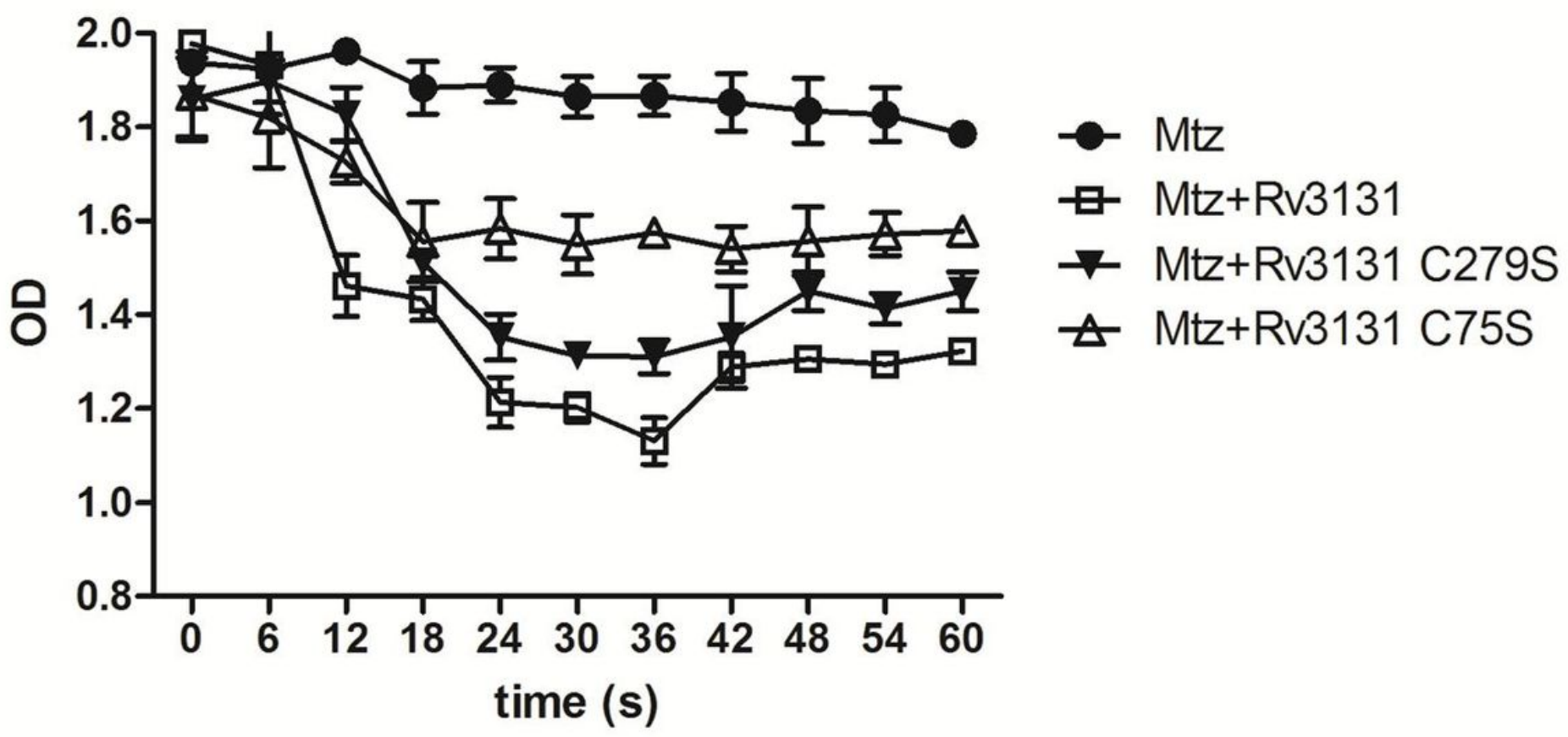

Figure 5

The nitroreductase activities of wild-type Rv3131 and mutants. 